\title{
Ventricular Tachycardia Detection Using Bipolar Electrogram Analysis is Site Specific
}

\author{
LORENZO A. DICARLO, JANICE M. JENKINS, CHIH-MING J. CHIANG, \\ STUART A. WINSTON, MICHAEL J. SILKA, and KATHY MATNEY \\ From the Medical Computing Laboratory, Department of Electrical Engineering and Computer \\ Science, College of Engineering, and School of Medicine, University of Michigan, and the \\ Michigan Heart and Vascular Institute and Cardiac Electrophysiology Laboratory at St. Joseph \\ Mercy Hospital, Ann Arbor, Michigan
}

DiCARLO, L.A., ET AL.: Ventricular Tachycardia Detection Using Bipolar Electrogram Analysis is Site Specific. While algorithms for bipolar intraventricular electrogram analysis have potential use in complementing rate criteria for ventricular tachycardia (VT) detection by implantable antitachycardia devices, the sensitivity of such algorithms to the intracavitary site of electrogram detection has not been determined. In this study, unfiltered $(1-500 \mathrm{~Hz})$ electrograms were recorded from a bipolar electrode catheter initially positioned at the right ventricular (RV) apex (site 1) of 12 patients during sinus rhythm (SR1) and during induced monomorphic VT (VT1). Sinus rhythm (SR2) and the identical VT (VT2) were recorded a second time after repositioning the same electrode catheter within the RV apex (site 2) 7-44 mm (mean $\pm S D=15 \pm 10$ ) from its original site. The data were digitized at 1,000 Hz. Templates from SR 1 and SR2, respectively, were compared subsequently with individual intraventricular electrograms from 15-25 sec passages of SR1 and VT1 and SR2 and VT2, respectively, using correlation waveform analysis. At site 1, the mean patient correlation coefficient ranged from 0.982-0.998 during SR1 and 0.062-0.975 during VT1. At site 2, the mean patient correlation coefficient ranged from $0.995-0.998$ during SR2 and 0.113-0.983 during VT2. Using a correlation threshold of 0.9, VT was differentiated from SR in 11/12 patients (91\%) overall: $8 / 12$ patients (67\%) at site $1,9 / 12$ patients ( $75 \%$ ) at site 2 , and $6 / 12$ patients $(50 \%)$ at both sites. Thus, while discrimination of VT from SR is feasible with morphological analysis of bipolar right ventricular intracavitary electrograms, the accuracy of bipolar intraventricular electrogram analysis may depend upon intracavitary electrode location in selected patients. (PACE, Vol. 15, November, Part II 1992)

arrhythmia, antitachycardia devices, cardioverter-defibrillator, ventricular tachycardia

\section{Introduction}

Among the major advances proposed for antitachycardia device technology has been the development of devices for transvenous device implantation. ${ }^{1,2}$ Such an approach utilizes intracardiac electrodes for tachycardia detection and therapy. Because of the known limitations of utilizing rate and rate variations alone by antitachycardia devices for discriminating sinus tachycardia and su-

Address for reprints: Lorenzo A. DiCarlo, M.D., Reichert Health Building, R-3003, PO Box 994, Ann Arbor, MI 48106. Fax: (313) 434-0141. praventricular tachycardias from ventricular tachyarrhythmias, complementary algorithms that utilize the time-domain or frequency-domain characteristics of intracavitary electrogram have been developed. ${ }^{3-B}$

Whether intracavitary electrode catheter location might have an impact upon the accuracy of morphological methods of ventricular electrogram analysis has never been determined. The purpose of this study, therefore, was to assess the impact of intracavitary electrode location upon: (1) bipolar intraventricular electrogram morphology; and (2) the discrimination of ventricular tachycardia (VT) from sinus rhythm. 


\section{Methods}

\section{Electrophysiology Study}

Electrograms were recorded during routine cardiac electrophysiological studies for VT analysis, using a technique described previously. ${ }^{7}$ The patient population consisted of 11 men and 1 woman (ages $44-86$ years; mean $=61$ years). All 12 patients had coronary artery disease with previous myocardial infarction. The patients were studied in a supine position and in a fasting, postabsorptive state. After sedation with 1-3 mg of intravenous medazolam and $1 \%$ lidocaine for local anesthetic, one 7-French and two 6-French sidearm sheaths (Cordis Corp., Miami, FL, USA) were positioned in the right femoral vein using the Seldinger technique. Each patient received 50 units/ $\mathrm{kg}$ of heparin intravenously as a bolus. Three 6French quadrapolar electrode catheters (USCI Division, C.R. Bard Inc., Billerica, MA, USA) with an interelectrode distance of $1 \mathrm{~cm}$ were introduced and advanced under fluoroscopic guidance. One electrode catheter was positioned in the high right atrium or right atrial appendage. One electrode catheter was positioned in the right ventricular apex. After confirmation of VT by recording of the His-bundle near the tricuspid annulus by the third electrode catheter, this catheter was positioned in the right ventricular apex (site 1), providing two separate electrode catheters for right ventricular apex pacing and recording, respectively. In addition to intracardiac atrial, His-bundle, and ventricular electrograms three electrocardiographic limb leads (V1, I, and III) were also recorded continuously during the study.

Programmed ventricular stimulation was performed using a previously described protocol. ${ }^{9}$ Following induction and termination of monomorphic VT, fluoroscopy was performed in an arc of $180^{\circ}$ to determine the maximum distance between the distal electrodes of the two right ventricular electrode catheters. Cineventriculography was used to record their respective positions. While maintaining the position of the pacing electrode catheter, the recording electrode catheter was then repositioned under fluoroscopy from its initial location (site 1) to another location within the right ventricular apex (site 2). Fluoroscopy was repeated in an arc of $180^{\circ}$ to determine the maximum distance between the distal electrodes of the two right ventricular electrode catheters. Cineventriculography was repeated, subsequently, to record their respective positions. Programmed ventricular stimulation was repeated and the same monomorphic VT was reinduced.

After completion of electrophysiological testing, the individual cineventriculograms that demonstrated maximum separation of the electrode catheters at sites 1 and 2 were measured and the distance in millimeters was corrected for fluoroscopic magnification using the measured diameter of the distal tip of the subsequently withdrawn electrode catheters as a reference.

\section{Data Acquisition}

Twelve-lead electrograms were recorded during sinus rhythm and induced monomorphic ventricular after positioning of the recording electrode catheters at sites 1 and 2. Bipolar intraventricular electrograms were recorded continuously on FM magnetic tape (Hewlett-Packard 3968A, San Diego, CA, USA) at a tape speed of $3.75 \mathrm{in} / \mathrm{sec}(9.5$ $\mathrm{cm} / \mathrm{sec}$ ) (bandwidth of $0-1250 \mathrm{~Hz}$ ) after signal amplification and filtering of $1-500 \mathrm{~Hz}$ (Honeywell Electronics-for-Medicine, Pittsburgh, PA, USA). Amplifier gain and filter settings were held constant during the entire recording procedure.

Passages of bipolar intraventricular electrograms during sinus rhythm and sustained monomorphic VT were concurrently digitized offline on an IBM-PC compatible (Advanced Logic Research 386/2, Irvine, CA, USA). A data acquisition system (Qua-Tech SAC-12, Akron, OH, USA), with a CODAS (Dataq Instruments, Akron, $\mathrm{OH}$, USA) waveform sampling and display system digitized data at a frequency of $1,000 \mathrm{~Hz} / \mathrm{channel}$. Tracings of the surface lead V1, I, and III electrocardiograms and bipolar electrograms were obtained during digitization using strip chart recorders (Gould Brush 481, Gould Inc. Recording Systems Div., Cleveland, OH, USA). The digitized versions of these signals were simultaneously viewed on the computer monitor.

\section{Method of Analysis}

From the site 1 and site 2 recordings of each patient, a 15-25 sec passage of sinus rhythm was digitized bipolar electrogram configurations. A second passage consisting of 15-25 sec of VT was 
also digitized. From each of the 2 sites, a ventricular'depolarization template was created for each patient by signal-averaging the first four cycles of sinus rhythm. The remainder of the sinus rhythm passage and the entire VT recording were subsequently analyzed using the sinus rhythm template.

Correlation waveform analysis, which is independent of electrogram amplitude and baseline fluctuations, was used as a statistical measure of the similarity, or dissimilarity, of the waveform under analysis when compared to the sinus rhythm template. ${ }^{10}$ The correlation coefficient was defined as:

$$
\rho=\frac{\sum_{k=1}^{N}\left(t_{k}-t\right)\left(S_{k}-s\right)}{\sqrt{\sum_{k=1}^{N}\left(t_{k}-t\right)^{2} \sum_{k=1}^{M}\left(S_{k}-s\right)^{2}}}
$$

where $t_{k}=$ the template points, $S_{k}=$ the signal points to be processed, $t=$ the average value of the template points, $s=$ the average value of the signal points, $N=$ the number points in the template, and $\rho=$ the index of merit. The value of the correlation coefficient, therefore, fell between \pm 1 such that identical waveforms had a value +1 , waveforms that were inverses of the template had a value of -1 , and all other dissimilar waveforms had values between +1 and -1 .

A patient-specific window size was chosen to define the ventricular depolarization portion of the waveform alone and to exclude local repolarization and injury current caused by the temporary catheter. The end of depolarization was defined as the instant at which repolarization appeared to begin in the bipolar electrogram. The beginning of depolarization was defined at the first deviation from baseline.

A software trigger was used to determine the position of ventricular depolarizations. This trigger consisted of a digital differentiator to determine the presence of ventricular depolarization. The method accounted for possible trigger jitter by finding the largest $\rho$ within an $11 \mathrm{msec}$ window surrounding the initial point specified by the differentiator. This maximum $\rho$ was reported as the value for each depolarization, and the range and mean of values for each analyzed passage were reported..$^{8}$ Irregular ventricular depolarizations during sinus rhythm, due to premature atrial or ventricular depolarizations not eliminated by the au- tomatic triggering process, were removed from the analysis manually.

\section{Results}

The difference in electrode location between site 1 and site 2 within the right ventricular apex ranged from 7-44 mm (mean $\pm S D=15 \pm 10$ $\mathrm{mm}$ ).

At site 1, the mean patient correlation coefficient ranged from $0.982-0.998$ during sinus rhythm (SR1) and 0.062-0.975 during induced monomorphic VT (VT1). At site 2, the mean patient correlation coefficient ranged from $0.995-0.998$ during SR2 and 0.113-0.983 during VT2.

Using a correlation threshold of 0.9 , VT was differentiated from SR in $11 / 12$ patients (91\%) overall: $8 / 12$ patients $(67 \%)$ at site $1,9 / 12$ patients $(75 \%)$ at site 2 , and $6 / 12$ patients $(50 \%)$ at both sites.

No correlation was found between the distance separating site 1 from site 2 and the ability to distinguish VT from SR using mean correlation coefficients and a threshold of 0.9 as a discriminator.

\section{Discussion}

Correlation waveform analysis has been shown previously to be effective in the discrimination of ventricular electrograms of normal sinus rhythm from those of VT, ventricular fibrillation, and paroxysmal bundle branch block of supraventricular origin. ${ }^{7,11,12}$ It has been incorporated recently into a real-time system of automated intracavitary atrial and ventricular electrogram analysis for arrhythmia diagnosis. ${ }^{13}$ Although it is computationally demanding, correlation waveform analysis has remained in our laboratory a reliable, time-domain "standard" for comparison of alternative algorithms developed to characterize intracavitary electrograms. ${ }^{7,8.14}$

The results of the present study confirm previous observations that discrimination of VT from $\mathrm{SR}$ is feasible with correlation waveform analysis of bipolar right ventricular intracavitary electrograms. The findings of the present study, however, would suggest that the accuracy of bipolar 
intraventricular electrogram analysis may depend upon intracavitary electrode location in selected patients.

There are several factors that could account for the findings of the present study: (1) the muscle bundles of ventricular myocardium are not uniformly parallel, resulting in anisotropy even in normal ventricles; (2) anisotropy is further magnified if structural changes in muscle fibers and their intercalated discs have occurred as a result of heart disease; (3) while the waveform of ventricular activation is three-dimensional, bipolar detection of ventricular activation is only two-dimensional because only the projection of the activation onto the axis of the bipole is recorded; and (4) although bipolar recordings of ventricular electrograms are advantageous because of noise elimination, the size of the bipolar lead field is inversely proportional to the cubed distance of the activation source from the bipole. As a consequence, the field

\section{References}

1. Moore SL, Maloney JD, Edel TB, et al. Implantable cardioverter-defibrillator implanted by nonthoracotomy approach: initial clinical experience with the redesigned transvenous lead system. PACE $1991 ; 14: 1865-1869$.

2. Saksena S, Mehta D, Krol RB, et al. Experience with a third generation implantable cardioverterdefibrillator. Am J Cardiol 1991; 67:1375-1384.

3. Davies DW, Wainwright RJ, Tooley MA, et al. Endocardial electrogram analysis for the automatic recognition of ventricular tachycardia. Circ 1985; 72:III-474.

4. Langberg JJ, Gibb WJ, Auslander DM, et al. Identification of ventricular tachycardia with the use of the morphology of the endocardial electrogram. Circ 1988; 77:1363-1369.

5. Pannizzo F, Furman S. Frequency spectra of ventricular and sinus rhythm in human intracardiac electrograms-application to tachycardia detection for cardiac pacemakers. IEEE Trans Biomed Eng 1988; 35:421-425.

6. Ropella KM, Baerman JM, Sahakian AV, et al. Differentiation of ventricular tachyarrhythmias. Circ 1990; 82:2035-2043.

7. Lin D, DiCarlo LA, Jenkins JJ. Identification of ventricular electrograms: Analysis of time and frequency domain patterns. PACE 1988; 11: 1592-1606.

8. Throne RD, Jenkins JJ, DiCarlo LA. A comparison of four new time-domain techniques for discriminating monomorphic ventricular tachycardia from of electrogram detection has the disadvantage of being small relative to the global waveform of ventricular activation. Small changes in the orientation of a small bipole relative to the global electrical wave of ventricular activation could result in significant changes in the bipolar waveform that is subsequently detected.

Previous studies have reported improved discrimination of VT from sinus rhythm with correlation waveform analysis when unipolar electrograms are compared to bipolar electrograms in selected patients. ${ }^{15}$ Further study will be necessary to determine what impact changes in intracavitary electrode location may have on these observations.

Acknowledgments: The authors wish to thank Beth Benedict, RT(R), Judy Jaffke, RT(R), and Pamela Parent, RN, for their technical assistance in the Cardiac Electrophysiology Laboratory.

sinus rhythm using ventricular waveform morphology. IEEE Trans Biomed Eng 1991; 38: 561-570.

9. DiCarlo LA, Susser F, Winston SA. The role of beta-blockade therapy for ventricular tachycardia induced with isoproterenol: A prospective analysis. Am Heart J 1990; 120:1347-1355.

10. Blum JR, Rosenblatt JI. Probability and Statistics. Philadelphia, PA, W.B. Saunders Co., 1972, p. 193.

11. DiCarlo LA, Jenkins JM, Winston SA, et al. Differentiation of ventricular tachycardia from ventricular fibrillation using intraventricular electrogram morphology. Am J Cardiol 1992 (In Press).

12. Throne RD, DiCarlo LA, Jenkins JM, et al. Paroxysmal bundle branch block of supraventricular origin: A possible source of misdiagnosis in detecting ventricular tachycardia using time-domain analysis of intraventricular electrograms. PACE 1990; 13:453-468.

13. Chiang CJ, Jenkins JM, DiCarlo LA. Realtime automatic detection of complex arrhythmias using rate augmented by intraatrial and intraventricular electrogram analysis. (abstract) PACE 1992; 15:529.

14. Throne RD, Jenkins JM, DiCarlo LA. Intraventricular electrogram analysis for ventricular tachycardia detection: Statistical validation. PACE 1990; 13:1596-1601.

15. Greenhut SE, DiCarlo LA, Jenkins JM, et al. Identification of ventricular tachycardia using intraventricular electrograms: A comparison of unipolar versus bipolar waveform analysis. PACE 1991; 14: 427-433. 
This document is a scanned copy of a printed document. No warranty is given about the accuracy of the copy. Users should refer to the original published version of the material. 